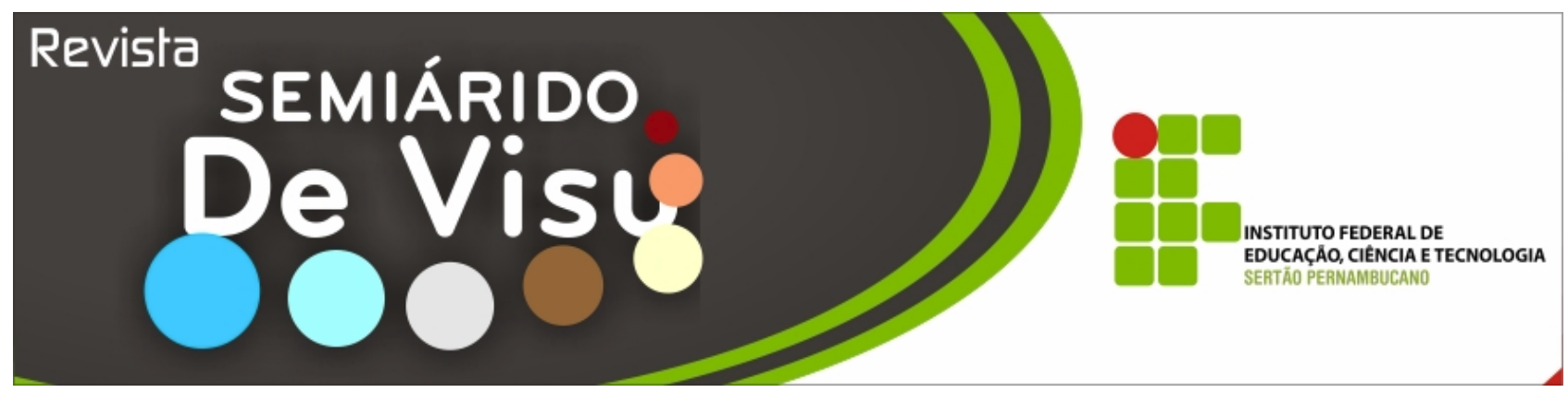

\title{
Caracterização e obtenção de farinha do resíduo gerado no processo industrial de clarificação do suco de acerola
}

\author{
Ana Carolina Dias Braga ${ }^{1}$, Marcos dos Santos Lima ${ }^{1 *}$, Luciana Cavalcanti de Azevedo ${ }^{1}$, Marta \\ Eugênia Cavalcanti Ramos ${ }^{1}$ \\ ${ }^{1}$ Instituto Federal do Sertão Pernambucano - Campus Petrolina, Rodovia BR 407 Km 08, Jardim São Paulo S/N \\ Petrolina - PE, CEP 56314-520, Fone (87) 3863-2330. Emails: carolbragga1@,hotmail.com.br, \\ *marcos.santos@ifsertao-pe.edu.br, luciana.cavalcanti@,ifsertao-pe.edu.br, marta.eugenia@ifsertao-pe.edu.br \\ *Autor para correspondência
}

\begin{abstract}
RESUMO: O processamento de frutas pelas indústrias alimentícias tem gerado uma grande quantidade de resíduos, os quais podem apresentar potencial para serem utilizados como fontes alternativas de nutrientes, principalmente, no desenvolvimento de novos produtos. O presente trabalho consistiu na caracterização físico-química e nutricional dos resíduos de acerola verde e madura a das farinhas obtidas pós-secagem, resultantes do processo industrial de suco de acerola clarificado e concentrado. Os resíduos frescos de acerola apresentaram boa quantidade de nutrientes,

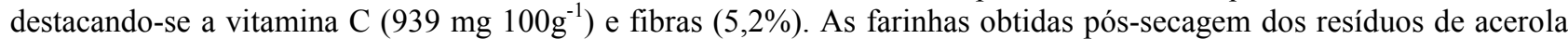
verde e madura, apresentaram alto valor nutricional, destacando-se a elevada concentração de vitamina $\mathrm{C}$ (em torno de $\left.2.500 \mathrm{mg} 100 \mathrm{~g}^{-1}\right)$ e fibras $(35 \%)$, evidenciando o bom potencial de utilização destas farinhas para o enriquecimento de produtos alimentícios.
\end{abstract}

Palavras-chave: Reaproveitamento, vitamina C, fibra alimentar.

\section{Characterization and extraction of flour from industrial waste generated during clarification of acerola juice}

\begin{abstract}
Fruit processing in fruit derived products industries generates a lot of residuals, which may have a potential use as alternative source of nutrients, mainly to develop new products. This work deals on the physical, chemical and nutritional characteristics of the residuals of unripe, ripe acerola fruit and its flour obtained after drying of the residuals of fruit, which has been used for the production of clarified and concentrated acerola juice. Residuals of fresh acerola has high concentration of nutrients, mainly $\mathrm{C}$ vitamin $\left(939 \mathrm{mg} 100 \mathrm{~g}^{-1}\right)$ and fiber (5.2\%). Flour of dried

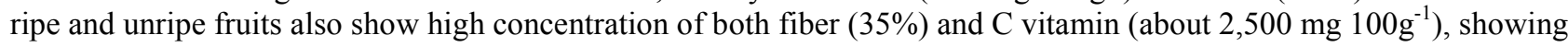
an interesting potential of this flour for the enrichment of food products.
\end{abstract}

Keywords: Reuse, $\mathrm{C}$ vitamin and dietary fiber. 
Ana Carolina Dias Braga et al.

\section{Introdução}

acerola ou cereja das Antilhas
(Malpighia glabra L.) é
originaria da América tropical, sendo amplamente cultivada nas regiões Nordeste e Sudeste do Brasil. Os elevados teores de vitamina $\mathrm{C}$, ou ácido ascórbico, naturalmente encontrados neste fruto, têm favorecido a ampliação de oportunidades para o seu cultivo, processamento e comercialização (AGOSTINI-COSTA et al., 2003).

Sabe-se que durante o processamento de alguns frutos ocorrem perdas de ácido ascórbico, variando de acordo com o tipo de processo e equipamentos utilizados (MATSUURA \& ROLIM, 2003). No entanto, mesmo após o processamento da acerola, os produtos gerados retêm um alto conteúdo desta vitamina (SEMENSATO, 1997).

$A$ vitamina $C$ inibe a síntese química de nitrosaminas, importante fator de risco para câncer do estômago. Estudos epidemiológicos e ensaios clínicos sugerem que uma ingestão diária de vitamina $\mathrm{C}$ maior do que a recomendada pelo Ministério da Saúde pode reduzir o risco de doenças crônicas como problemas cardíacos e câncer, especialmente quando combinados com a ingestão de vitamina $\mathrm{E}$ (HATHCOCK, 1997).

Os resíduos desprezados pelas indústrias alimentícias também podem ser utilizados como fontes alternativas de fibras, pois é conhecido que várias cascas, folhas $\mathrm{e}$ talos possuem elevadas concentrações de fibras dietéticas, que possuem atuação na prevenção de doenças cardiovasculares e gastrointestinais (PEREIRA et al., 2003). As fibras alimentares apresentam efeitos fisiológicos importantes, e uma alternativa ao aumento de seu consumo pela população é a utilização de resíduos agroindustriais como matéria-prima para a produção de alguns alimentos enriquecidos (OLIVEIRA et al., 2003).

A utilização de subprodutos da acerola (semente triturada e polpa, após a retirada do suco) na formulação de alimentos exige o aprofundamento de pesquisas no sentido de se conhecer o valor nutritivo dos mesmos, já que eles representam entre 15 e $41 \%$ do volume total de toda acerola processada (VASCONCELOS et al., 2002).

Uma das formas de aproveitamento de subprodutos da indústria alimentícia é a elaboração de farinha. A qualidade de farinhas pode ser avaliada por diversas características como umidade, matéria mineral, lipídios, proteínas entre outros (CIACCO \& CHANG, 1986). Estas propriedades refletem o processo de beneficiamento e podem ser empregadas para avaliar a qualidade tecnológica ou nutricional do produto (DUTCOSKY, 1995). Portanto, o presente estudo tem como objetivo a caracterização físico-química do resíduo industrial de acerolas verde e madura, e a obtenção e caracterização da farinha após a aplicação de secagem.

\section{Material e métodos}

\section{Local de execução e coleta da amostra}

O experimento foi realizado no Laboratório Experimental de Alimentos (LEA) do Instituto Federal do Sertão Pernambucano (IF SERTÃO - PE), situado na rodovia BR 407, km 08, Jardim São Paulo, Petrolina-PE. Os resíduos foram coletados no decanter (modelo SC 4506076) utilizado no processo de clarificação de suco de acerola pela empresa Niagro - Nichirei do Brasil Agrícola Ltda, localizada no distrito industrial de PetrolinaPE. A coleta da matéria-prima foi realizada nos meses de março e abril de 2009, sendo composta por resíduos mistos das variedades: Okinawa, Sertaneja, Flor Branca, Costa Rica, Junko e Nik, plantadas na região do Vale Submédio do Rio São Francisco. As amostras foram acondicionadas em sacos de polietileno e transportadas ao IF SERTÃO - PE Campus Petrolina, onde foram congeladas em freezer horizontal e mantidas à temperatura de $-18^{\circ} \mathrm{C}$ até a realização das análises físico-químicas. 
Ana Carolina Dias Braga et al.

No processo de obtenção do suco de acerola clarificado, a acerola foi despolpada, pasteurizada a $95^{\circ} \mathrm{C}$ por 12 segundos e resfriada até $45^{\circ} \mathrm{C}$, seguiu para tanques de enzimação onde foi adicionada a enzima poligalacturonase. Após enzimação, a polpa passou pelo decanter onde foi separado o suco da polpa, gerando um resíduo sólido que equivaleu a $10 \%$ do volume do total da polpa inicial de acerola processada.

\section{Análises físico-químicas}

Foram realizadas analises em triplicata nas amostras dos resíduos frescos de acerola verde e madura, e nas farinhas obtidas pela secagem dos resíduos. Foram feitas determinações de sólidos solúveis, vitamina $\mathrm{C}$, acidez titulável, cinzas, umidade, gorduras totais, proteínas e açúcares redutores, utilizando a metodologia descrita pelo Instituto Adolfo Lutz (2008), e fibra bruta pela metodologia descrita por Cecchi (1999).

\section{Secagem do resíduo de acerola para obtenção da Farinha}

Os resíduos de acerola verde (RAV) e madura (RAM) foram pesados, acondicionados em bandejas de aço inoxidável teladas, colocados em secador elétrico (marca Pardal, Brasil) e secaram na temperatura de $65^{\circ} \mathrm{C}$ com circulação forçada de ar, até peso constante. Após a secagem os resíduos foram acondicionados em potes de polietileno com tampa, permanecendo à temperatura ambiente até o momento da realização das análises físico-químicas. Os resíduos secos (farinha) foram codificados como FRAV e FRAM, para as farinhas do resíduo de acerola verde e madura, respectivamente.

\section{Análise estatística}

O experimento foi conduzido inteiramente ao acaso, onde os tratamentos foram compostos pelos resíduos frescos de acerola verde (RAV) e madura (RAM), e as respectivas farinhas obtidas da secagem dos resíduos frescos (FRAV e FRAM), com cinco repetições, onde as repetições corresponderam às amostras coletadas em diferentes datas. As médias obtidas da caracterização dos resíduos frescos e das farinhas foram comparadas entre si pelo teste $t$ a $5 \%$ de probabilidade, utilizando o programa estatístico SISVAR 4.2 (Build 39).

\section{Resultados e discussão}

Os resultados das análises físicoquímicas dos resíduos de acerola verde e madura estão apresentados na tabela 1 .

Foram encontrados valores de sólidos solúveis nas amostras dos resíduos RAV e RAM na ordem de 2,35 e 4,96\%, respectivamente. No RAM a concentração destes sólidos foi maior que no RAV, provavelmente devido ao processo de maturação, que com o avanço, promove $o$ acúmulo de sólidos solúveis nos frutos (CHITARRA \& CHITARRA, 2005). Nogueira et al. (2002) mencionaram valores de sólidos solúveis em acerolas entre 7,0 e $8,73 \%$ em função da época de colheita e do estádio de maturação. 
Ana Carolina Dias Braga et al.

Tabela 1. Caracterização dos resíduos de acerola (Malpighia glabra L.) verde (RAV) e madura (RAM), extraídos do processo industrial de clarificação do suco.

\begin{tabular}{|c|c|c|}
\hline COMPONENTE & RAV & $\overline{\text { RAM }}$ \\
\hline Sólidos solúveis \% & $2,35 \mathrm{~b}$ & $4,96 \mathrm{a}$ \\
\hline Vitamina C (mg 100g $\left.{ }^{-1}\right)$ & $730 \mathrm{a}$ & 939 a \\
\hline Acidez titulável (\% ácido málico) & $0,61 \mathrm{a}$ & $0,40 \mathrm{~b}$ \\
\hline $\mathrm{pH}$ & $3,12 \mathrm{~b}$ & $3,27 \mathrm{a}$ \\
\hline Umidade $\%$ & 89,9 a & $85,5 \mathrm{a}$ \\
\hline Cinzas \% & $0,22 \mathrm{~b}$ & $0,4 \mathrm{a}$ \\
\hline Gorduras totais $\%$ & $2,32 \mathrm{a}$ & $2,32 \mathrm{a}$ \\
\hline Fibra bruta $\%$ & $4,2 \mathrm{a}$ & $5,2 \mathrm{a}$ \\
\hline Açúcares redutores (\% glicose) & $1,8 \mathrm{~b}$ & $2,38 \mathrm{a}$ \\
\hline Proteínas \% & ND & ND \\
\hline
\end{tabular}

A concentração de vitamina $\mathrm{C}$ em ambos os resíduos variou de 730 a $939 \mathrm{mg}$ $100 \mathrm{~g}^{-1}$ para RAV e RAM respectivamente, mesmo com o processo de lavagem realizado na extração do suco. Estes valores foram considerados elevados, pois Nogueira et al. (2002) mencionaram valores de vitamina $\mathrm{C}$ em

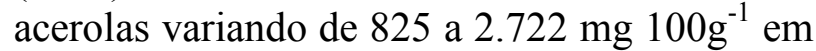
função da variedade, estádio de maturação e época de colheita. Desta forma, pode-se afirmar que estes resíduos consistem numa boa fonte de vitamina $\mathrm{C}$.

$\mathrm{O}$ resíduo RAV apresentou maior acidez titulável $(0,61 \%)$ que o resíduo RAM $(0,4 \%)$. Com relação ao $\mathrm{pH}$, o RAV obteve valor de 3,12 e o RAM 3,27, resultado normal visto que com o processo de maturação dos frutos há síntese de sólidos solúveis e diminuição de ácidos pelo processo de respiração (CHITARRA \& CHITARRA, 2005). França \& Narain (2003) encontraram maiores valores de acidez titulável e menores valores de $\mathrm{pH}$ em acerolas "de vez" comparando-se com as acerolas maduras, independente da variedade estudada.

Os valores de umidade para o RAV e RAM (89,9\% e 85,5\% respectivamente) foram considerados elevados, uma vez que podem favorecer a multiplicação microbiana e reações químicas de degradação, reduzindo o tempo de vida útil destes resíduos.
A quantidade de cinzas no RAM $(0,4 \%)$ foi maior que no RAV $(0,22 \%)$, possivelmente devido à acerola madura possuir maior quantidade de elementos minerais em decorrência do processo de maturação. Os resultados obtidos estão próximos da faixa de valores encontrada por outros autores em acerolas frescas, que em média, se apresentaram entre 0,33 e $0,37 \%$ (FRANÇA \& NARAIN, 2003).

Ambos os resíduos de acerola obtiveram valores de gorduras totais equivalentes $(2,32 \%)$. Os resíduos RAM e RAV apresentaram quantidade de fibra bruta de 5,2 e $4,2 \%$, respectivamente, sendo considerada uma quantidade significativa de fibras se comparado com frutas como a goiaba vermelha $(6,2 \%)$, ameixa $(2,4 \%)$ e maçã $(2,0 \%)$ (TACO, 2011). Segundo Brasil (1998), um alimento pode ser considerado como fonte de fibra

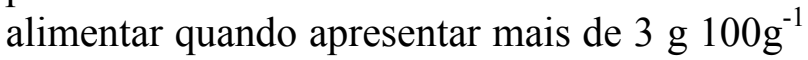
(base integral) para alimentos sólidos e 1,5 g $100 \mathrm{~mL}^{-1}$ para alimentos líquidos.

Os resíduos apresentaram valores de açúcares redutores em torno de 2,0\% para o RAM e RAV, resultado esperado, uma vez que o resíduo foi lavado no processo de obtenção do suco clarificado. França \& Narain (2003) mencionaram valores de açúcares redutores entre 3,0 e $4,0 \%$ em acerolas de diferentes variedades e estádios de maturação. 
Ana Carolina Dias Braga et al.

\section{Farinha dos resíduos de acerola}

Os resultados analíticos das farinhas obtidas dos resíduos de acerola verde e madura estão apresentados na tabela 2.

Os valores de vitamina $\mathrm{C}$ encontrados na farinha do resíduo de acerola verde (FRAV) e madura (FRAM) demonstram que houve uma concentração desta vitamina com a retirada de água no processo de secagem, passando de 730

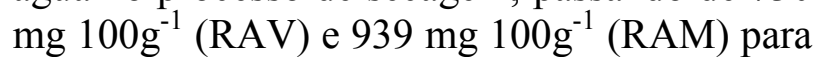
$2231 \mathrm{mg} 100 \mathrm{~g}^{-1}$ e $2571 \mathrm{mg} 100 \mathrm{~g}^{-1}$ nas farinhas FRAV e FRAM, respectivamente. Considerando-se a necessidade de ingestão diária (IDR) de $60 \mathrm{mg}$ de vitamina $\mathrm{C}$ para adultos (BRASIL, 1998), fica constatado que a farinha do resíduo de acerola é uma fonte considerável desta vitamina. Diversos autores sugerem um (IDR) de $200 \mathrm{mg}$ de vitamina C (AUSMAN, 1999; LEVINE et al., 1999), o que, com apenas oito gramas da farinha do resíduo de acerola, já se atenderia esta recomendação.

Os valores de vitamina $\mathrm{C}$ encontrados em FRAV e FRAM foram muito superiores aos encontrados em farinha de banana verde (15 $\left.\mathrm{mg} 100 \mathrm{~g}^{-1}\right)$ e nos resíduos secos de bagaço de

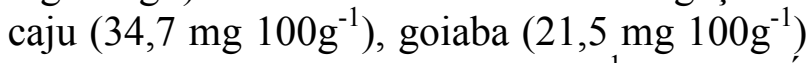
e casca de maracujá $\left(11,8 \mathrm{mg} 100 \mathrm{~g}^{-1}\right)$ (CHISTÉ et al., 2006; UCHOA et al., 2008). A elevada concentração de vitamina $\mathrm{C}$ encontrada nas farinhas do resíduo de acerola evidencia o seu potencial de utilização na alimentação humana e no enriquecimento de produtos alimentícios.

Em relação à umidade, todas as amostras apresentaram-se dentro dos padrões exigidos pela legislação brasileira, que estabelece um máximo de $13 \%$ para farinhas (BRASIL, 1995). Os valores obtidos foram $5,12 \%$ para FRAV e 5,51\% para FRAM.

Tabela 2. Caracterização das farinhas dos resíduos de acerola (Malpighia glabra L.) verde (FRAV) e madura (FRAM), extraídos do processo industrial de clarificação do suco.

\begin{tabular}{ccc}
\hline COMPONENTE & FRAV & FRAM \\
\hline Vitamina C $\left({\left.\mathrm{mg} 100 \mathrm{~g}^{-1}\right)}^{-1}\right.$ & $2231 \mathrm{a}$ & $2571 \mathrm{a}$ \\
Acidez titulável $(\%$ ácido málico) & $3,61 \mathrm{a}$ & $3,06 \mathrm{~b}$ \\
Umidade \% & $5,12 \mathrm{a}$ & $5,51 \mathrm{a}$ \\
Cinzas \% & $5,69 \mathrm{a}$ & $4,75 \mathrm{a}$ \\
Gorduras Totais \% & $13,48 \mathrm{a}$ & $12,93 \mathrm{a}$ \\
Fibra Bruta \% & $35,11 \mathrm{a}$ & $35,78 \mathrm{a}$ \\
Açúcares redutores $\%$ glicose) & $11,7 \mathrm{a}$ & $14,4 \mathrm{~b}$ \\
\hline
\end{tabular}

*Médias seguidas de letras iguais, na linha, não diferem entre si pelo teste $t$ a $5 \%$ de probabilidade.

Foram encontrados teores de lipídios em FRAV de 13,48\% e em FRAM (12,93\%). Os valores de açúcares redutores para FRAV e FRAM foram de $11,7 \%$ e $13,4 \%$ respectivamente, sendo estes valores, inferiores ao da farinha de batata-doce não extrusada (17,0\% de açúcares solúveis totais) (BORBA et al., 2005).

As farinhas dos resíduos de acerola apresentaram elevadas quantidades de fibra bruta $(35,1$ e $35,8 \%$ para FRAV e FRAM respectivamente), se comparado com a farinha da casca de manga Tommy Atkins (8,28\%), farinha de banana verde $(1,17 \%)$, farinha da casca da batata inglesa $(4,77 \%)$, e os pós alimentícios obtidos do bagaço de caju $(9,9 \%)$, bagaço de goiaba $(39,5 \%)$ e casca de maracujá (26,3\%) (AZEVEDO et al., 2008; BORGES et al., 2009; FERNANDES et al., 2008; UCHOA et al., 2008). 
Ana Carolina Dias Braga et al.

\section{Conclusões}

A avaliação da composição dos resíduos de acerola verde e madura, extraídos do processo de clarificação do suco, revelou que este subproduto possui um elevado valor nutricional. As farinhas dos resíduos de acerola verde e madura apresentaram maiores quantidades de nutrientes do que o resíduo fresco, destacando-se a alta concentração de vitamina $\mathrm{C}$ e fibras.

\section{Agradecimentos}

A Niagro - Nichirei Agrícola Ltda pelo apoio na execução deste trabalho.

\section{Referências}

AGOSTINI-COSTA, T. S.; ABREU, L. N.; ROSSETTI, A. G. Efeito do congelamento e do tempo de estocagem de polpa de acerola sobre o teor de carotenóides. Revista Brasileira de Fruticultura, v. 25, 2003.

AUSMAN, L. M. Recommendations for Vitamin C intake. Nutr. Ver. 1999; 57:222-9.

AZEVÊDO, L. C.; AZOUBEL, P. M.; SILVA, I. R. A.; ARAÚJO, A. J. de B.; OLIVEIRA, S. B. Caracterização Físico-Química da Farinha da Casca de manga cv. Tommy Atkins. In: CONGRESSO BRASILEIRO DE CIÊNCIA E TECNOLOGIA DE ALIMENTOS, 21; SEMINÁRIO LATINO AMERICANO E DO CARIBE DE CIÊNCIA E TECNOLOGIA DE ALIMENTOS, 15. Ciência e inovação para o desenvolvimento sustentável. Belo Horizonte: SBCTA, 2008.
BORBA, A. M.; SARMENTO, S. B. S.; LEONEL, M. Efeito dos parâmetros de extrusão sobre as propriedades funcionais de extrusados da farinha de batata-doce. Ciências Tecnologia de Alimentos. vol. 25 no.4 Campinas, 2005.

BORGES, A. M.; FERREIRA, J.; LUCENA, E. M. P. Caracterização da farinha de banana verde. Ciênc. Tecnol. Aliment., Campinas, 29(2): 333-339, abr.-jun. 2009

BRASIL. Ministério da Saúde. Secretaria de Vigilância Sanitária. Portaria $n^{\circ} 33$, de 13 de janeiro de 1998. Adota valores para a ingestão diária recomendada (IDR) de vitaminas, minerais e proteínas. Diário Oficial [da] República Federativa do Brasil, Brasília, DF, 16 jan. 1998.

BRASIL. Portaria $n^{0} 554$ de 30 de agosto de 1995. Diário Oficial. Brasília, Secretaria da Agricultura, do Abastecimento e Reforma Agrária. 1 Set., Seção 1.

CECCHI, H. M. Fundamentos Teóricos e práticos em análises de alimentos. Campinas, SP. Editora da UNICAMP. 1999. 211p.

CHAVES, M. C. V; GOUVEIA, J. P. G; ALMEIDA, F. A. C; LEITE, J. C. A; SILVA, F. L. H. Caracterização físico-química do suco de acerola. Revista de Biologia e Ciências da terra, v.4, n.2, 2004.

CHISTÉ, R. C.; COHEN, K. O.; MATHIAS, E. A.; JÚNIOR, A. G. A. R. Qualidade da Farinha de Mandioca do grupo seca. Ciênc. Tecnol. Aliment. vol. $26 \mathrm{n}^{\mathrm{0}} 4$ Campinas Oct./Dec. 2006.

CHITARRA, M. I. F.; CHITARRA, A. B. Póscolheita de frutos e hortaliças: fisiologia e manuseio. Lavras: ESAL/FAEP, 2005. 782p. 
Ana Carolina Dias Braga et al.

CIACCO, C. F. \& CHANG, Y. K. Tecnologia de massas alimentícias. São Paulo: Ícone, 1986, 127p.

DUTCOSKY, S. D. Desenvolvimento de tecnologia de fabricação de biscoitos e massas alimentícias isentos de glúten a partir da farinha de arroz. Curitiba, 1995. 158p. Dissertação (Mestrado em Tecnologia Química) - Universidade Federal do Paraná.

FERNANDES, A. F.; PEREIRA, J.; GERMANI, R.; OIANO-NETO, J. Efeito da substituição parcial da farinha de trigo por farinha de casca de batata (Solanum Tuberosum Lineu). Ciênc. Tecnol. Aliment. vol.28 suppl.0 Campinas Dec. 2008.

FRANÇA, V. C.; NARAIN, N. Caracterização química dos frutos de três matrizes de acerola (Malpighia emarginata D. C.). Ciênc. Tecnol. Alim., Campinas, 23(2): 157-160, maio-ago. 2003

GARCIA, D. C.; BARROS, A. C. S. A.; PESKE, S. T.; MENEZES, N. L. A secagem de sementes. Ciência Rural, Santa Maria, v.34, n.2, p.603-608, 2004.

GIUNTINI, E. B.; LAJOLO, F. M.; MENEZES, E. W. Potencial de fibra alimentar em países ibero-americanos: alimentos, produtos e resíduos. Arch. Latinoam. Nutr., Caracas, v. 53, n.1, p. 1-7, 2003.

HATHCOCK, J. N. Vitamins and minerals: efficacy and safety. Am J Clin Nutr 1997;66:427-37.

IAL - Instituto Adolfo Lutz (São Paulo). Métodos físico-químicos para análise de alimentos /coordenadores Odair Zenebon, Neus Sadocco Pascuet e Paulo Tiglea - São Paulo: Instituto Adolfo Lutz, 2008. 1020p. Versão eletrônica.

LEVINE, M.; RUMSEY, S. C.; DARUWALA, R. Criteria and recommendations for vitamin C intake. JAMA 1999; 281:1415-23.
LIMA, V. L. A.; MÉLO, E. A.; MACIEL, M. I. S. et al. Avaliação do teor de antocianinas em polpa de acerola congelada proveniente de frutos de 12 diferentes aceroleiras (Malpighia emarginata D. C.). Ciência e Tecnologia de Alimentos, Campinas, v.23, n.1, p.101-103, 2003.

MATSUURA, F. C. A. U.; ROLIM, R. B. Avaliação da adição de suco de acerola em suco de abacaxi visando à produção de um "blend" com alto teor de vitamina C. Revista Brasileira de Fruticultura, Cruz das Almas, v.24, n.1, p.138-141, 2002.

NOGUEIRA, R. J. M. C.; MORAES, J. A. P. V.; BURITY, H. A.; JUNIOR, J.F.S. Efeito do estádio de maturação dos frutos nas características físico-químicas de acerola. Pesq. agropec. bras., Brasília, v. 37, n. 4, p. 463470, abr. 2002

OLIVEIRA, L. F. et al. Aproveitamento alternativo da casca do maracujá-amarelo (Passiflora edulis F. Flavicarpa) para produção de doce em calda. Ciênc.Tecnol. Alim., Campinas, v.22, n.3, p. 1-60, 2002.

PEREIRA, F. M., CARVALHO, C. A., NACHTIGAL, J. C. Século XXI: Nova cultivar de goiabeira de dupla finalidade. Ver. Brás. Frutic., Jabuticabal, SP, v.25, n.3, p. 498-500, 2003.

SEMENSATO, L. R. Caracterização físicoquímica de frutos genótipos de acerola (Malpighia sp.), cultivados em Anápoles-GO, processamento e estabilidade de seus produtos. Goiânia, 1997. 74f. Dissertação (Mestrado em Agronomia) - Universidade Federal de Goiânia.

TACO - Tabela brasileira de composição de alimentos / NEPA - UNICAMP. 4.ed. rev. e ampl. Campinas: NEPA - UNICAMP, 2011. 161p. Disponível em < http://www.unicamp.br/nepa/taco/contar/taco 4 edicao_ampliada e revisada.pdf?arquivo $=\mathrm{tac}$ 
Ana Carolina Dias Braga et al.

o_4_versao_ampliada_e_revisada.pdf

acessado em 25 de outubro de 2011.

UCHOA, A. M. A.; COSTA, J. M. C.; MAIA, G. A.; SILVA, E. M. C.; CARVALHO, A. F. F. U.; MEIRA, T. R. Parâmetros FísicoQuímicos, Teor de Fibra Bruta e Alimentar de Pós Alimentícios Obtidos de Resíduos de Frutas Tropicais. Alimentar e Nutricional, Campinas, 15(2): 58-65, 2008.

VASCONCELOS, V. R.; NEIVA, J. N. M.; PIMENTEL, J. C. M. et al. Utilização de subprodutos do processamento de frutas na alimentação de caprinos e ovinos. In: VI SEMINÁRIO NORDESTINO DE PECUÁRIA - PECNORDESTE, Fortaleza-CE, Anais... Fortaleza: FAEC, 2002. p.83-99.

YAMASHITA, F.; BENASSI, M. T.; TONZAR, A. C.; MORIYA, S.; FERNANDES, J. G. Produtos de acerola: estudos da estabilidade de vitamina C. Ciência e Tecnologia de Alimentos, Campinas, v. 23, n. 1, p. 92-94, 2003. 